\title{
Improved Precision of Automatic Brain Volume Measurements in Patients with Clinically Isolated Syndrome and Multiple Sclerosis Using Edema Correction
}

\author{
(D).B.M. Warntjes, (D)A. Tisell, (D). Håkansson, D.P. Lundberg, and (i)J. Ernerudh
}

\begin{abstract}
BACKGROUND AND PURPOSE: The presence of edema will result in increased brain volume, which may obscure progressing brain atrophy. Similarly, treatment-induced edema reduction may appear as accelerated brain tissue loss (pseudoatrophy). The purpose of this study was to correlate brain tissue properties to brain volume, to investigate the possibilities for edema correction and the resulting improvement of the precision of automated brain volume measurements.
\end{abstract}

MATERIALS AND METHODS: A group of 38 patients with clinically isolated syndrome or newly diagnosed MS were imaged at inclusion and after 1, 2, and 4 years using an MR quantification sequence. Brain volume, relaxation rates $\left(R_{1}\right.$ and $\left.R_{2}\right)$, and proton density were measured by automated software.

RESULTS: The reduction of normalized brain volume with time after inclusion was $0.273 \%$ /year. The mean SDs were $0.508 \%, 0.526 \%$, $0.454 \%$, and $0.687 \%$ at baseline and 1,2 , and 4 years. Linear regression of the relative change of normalized brain volume and the relative change of $R_{1}, R_{2}$, and proton density showed slopes of $-0.198(P<.001), 0.156(P=.04)$, and $0.488(P<.001)$, respectively. After we applied the measured proton density as a correction factor, the mean SDs decreased to $24.2 \%, 4.8 \%, 33.3 \%$, and $17.4 \%$, respectively. The observed atrophy rate reduced from $0.273 \%$ /year to $0.238 \%$ /year.

CONCLUSIONS: Correlations between volume and $R_{1}, R_{2}$, and proton density were observed in the brain, suggesting that a change of brain tissue properties can affect brain volume. Correction using these parameters decreased the variation of brain volume measurements and may have reduced the effect of pseudoatrophy.

ABBREVIATIONS: $B P F=$ brain parenchymal fraction; $\mathrm{BPV}=$ brain parenchymal volume; $\mathrm{ICV}=$ intracranial volume; $\mathrm{NEDA}=$ no evidence of disease activity; $\mathrm{PD}=$ proton density; $R_{1}$ and $R_{2}=$ relaxation rates; $S_{R 1}, S_{R 2}$, and $S_{P D}=$ correlation slopes

B rain atrophy and accelerated brain volume loss are present early in the disease course of MS and correlate with the presence and development of physical disability and cognitive impairment. ${ }^{1}$ When assessing disease activity in MS, the term "no evidence of disease activity" (NEDA) is increasingly used, where NEDA-3 is defined by the combination of the following: 1) no relapses, 2) no brain MR imaging activity (no new or enlarging T2 lesions or gadolinium-enhancing lesions), and 3) no sustained

Received July 5, 2017; accepted after revision October 8.

From the Center for Medical Image Science and Visualization (J.B.M.W., A.T., P.L.); Division of Cardiovascular Medicine (J.B.M.W.) and Radiation Physics (A.T., P.L.), Department of Medical and Health Sciences; Department of Neurology (I.H.), Department of Clinical and Experimental Medicine; Department of Clinical Immunology and Transfusion Medicine (J.E.); and Department of Clinical and Experimental Medicine (J.E.), Linköping University, Sweden; and SyntheticMR AB (J.B.M.W.), Linköping, Sweden.

Please address correspondence to J.B.M. Warntjes, MD, Center for Medical Image Science and Visualization (CMIV), Linköping University, 58185 Linköping, Sweden;

e-mail: marcel.warntjes@cmiv.liu.se

http://dx.doi.org/10.3174/ajnr.A5476 disability worsening (Expanded Disability Status Scale progression). However, given the clinical importance of brain atrophy, it is desirable to expand the NEDA concept to NEDA-4, in which "no increased brain volume loss/brain atrophy rate" is added. ${ }^{2}$ Disease-modifying immunomodulatory treatment options in MS vary in efficacy and adverse effect profile. Personalized treatment for a patient with MS requires clinically feasible and reliable assessment of disease activity status at treatment initiation and treatment follow-up. Although there is not yet enough evidence to support the use of brain volume measures to monitor treatment response or for making treatment decisions in individual patients, brain volume loss is now often incorporated in large clinical trials of potential new MS drugs. ${ }^{3}$ Monitoring brain volume and brain volume changes with time in clinical practice, however, is often hampered by the additional time and effort burden of brain segmentation methods and their inherent precision. Moreover, disease activity induces changes in the intra- or extracellular water content, which, in turn, can lead to changes in total brain volume. Therefore, resolution of inflammatory edema after 
Table 1: Patient diagnoses, relapse status, and treatment status at baseline and at followup MRI

\begin{tabular}{lcccc}
\hline \multicolumn{1}{c}{$\begin{array}{c}\text { Clinical and } \\
\text { Laboratory Data }\end{array}$} & Baseline & 1 Year & 2 Years & 4 Years \\
\hline $\begin{array}{l}\text { Diagnosis (CIS/RRMS/PPMS) } \\
\text { Relapse within last 2 mo }\end{array} \quad 16 / 19 / 3$ & $10 / 25 / 3$ & $7 / 28 / 3$ & $5 / 30 / 3$ \\
$\quad 13 / 25$ & $3 / 35$ & $1 / 37$ & $2 / 36$ \\
$\quad$ before MRI (yes/no) & & & & \\
Treatment ${ }^{2}$ (No. of subjects) & & 17 & 18 & 18 \\
No DMT & 38 & 16 & 12 & 5 \\
Interferon- $\beta$ lb & 0 & 1 & 1 & 1 \\
Interferon- $\beta$ la & 0 & 0 & 0 & 2 \\
Dimethyl fumarate & 0 & 1 & 1 & 3 \\
Fingolimod & 0 & 3 & 6 & 9 \\
Natalizumab & 0 & &
\end{tabular}

Note:-CIS indicates clinically isolated syndrome; RRMS, relapsing-remitting MS; PPMS, primary-progressive MS; DMT, disease modifying therapy.

${ }^{a}$ In addition, 1 patient received temporary relapse-controlling corticosteroid treatment within 2 months prior to baseline.

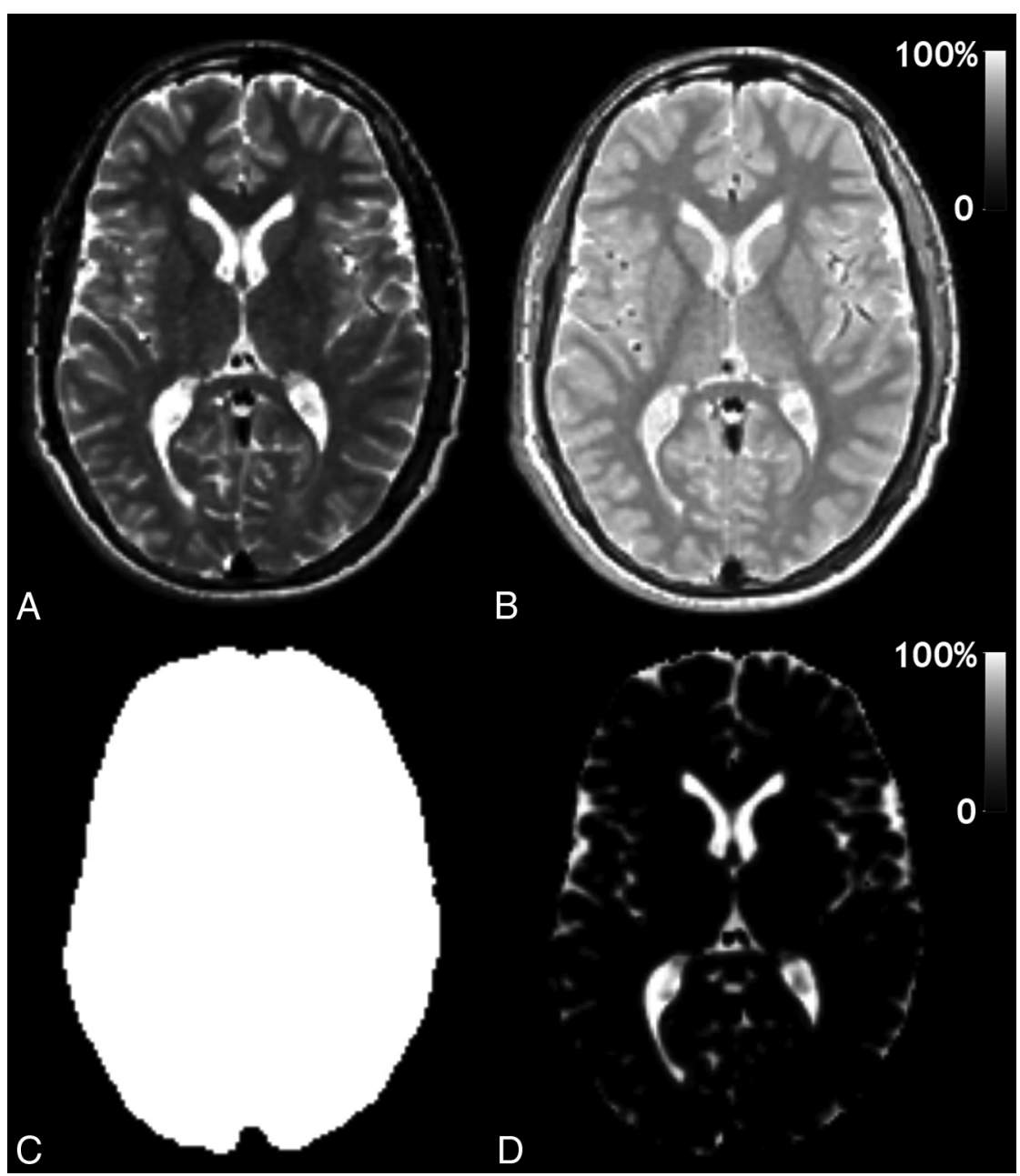

FIG 1. Typical output of the SyMRI software: $A$, Synthetic T2-weighted image of a section of the brain of one of the patients. This synthetic image was generated from the $R_{1}, R_{2}$, and PD maps in combination with a TE/TR $=100 / 4500$ ms. B, PD map on a scale of $0 \%-100 \%$, in which $100 \%$ corresponds to pure water at $37^{\circ} \mathrm{C}$. C, Automatically generated intracranial mask (ICV). D, Automatically generated CSF partial volume map on a scale of $0 \%-100 \%$. The brain parenchymal volume is calculated as the sum of all ICV minus the sum of all CSF. The brain parenchymal fraction is calculated as BPV/ICV.

a successful treatment may appear as atrophy, known as "pseudoatrophy," as previously reported in patients with early-onset MS. ${ }^{4,5}$ Likewise, an increase in disease activity may increase the total edema, visible only as diffuse hyperenhancement on T2- weighted or FLAIR images. This may lead to swelling of the brain, obscuring the progressing atrophy.

Recent progress in quantitative MR imaging permits measurement of physical properties of the brain, such as relaxation rates $\left(R_{1}\right.$ and $\left.R_{2}\right)$ and proton density (PD) in a reasonable scan time. ${ }^{6}$ Axonal damage, gliosis, inflammation, and edema are related to changes in these values. $^{7-12}$ Therefore, it can be expected that the mean values of $R_{1}, R_{2}$, and PD have a relationship with processes that may lead to global volume changes in the brain. SyntheticMR (SyMRI; SyntheticMR, Linköping, Sweden) is a brain segmentation tool with high precision, ${ }^{13-15}$ which is based on quantitative MR imaging. It therefore combines the ability to fully automatically measure brain volumes and simultaneously obtain values for mean $\mathrm{R}_{1}, \mathrm{R}_{2}$, and PD of brain tissue.

The aim of this study was to investigate the relation between brain volumes of patients with clinically isolated syndrome and those with MS using a fully automatic brain volume segmentation method and the mean $\mathrm{R}_{1}, \mathrm{R}_{2}$, and PD values in these brains. Provided that these 2 measures correlated, the second aim was to use $R_{1}, R_{2}$, and $P D$ as a correction factor for brain volume and investigate whether the precision of the brain segmentation method would improve.

\section{MATERIALS AND METHODS Subject Group}

The study included 44 patients (29 women) who were consecutively enrolled in a prospective longitudinal cohort study of patients with clinically isolated syndrome and those with newly diagnosed MS. All patients fulfilled the revised McDonald criteria for clinically isolated syndrome or MS at inclusion. ${ }^{16}$ Brain MR imaging was performed at baseline and at 1,2, and 4 years. Retrospectively, 1 subject was excluded due to severe hydrocephalus; for 5 subjects, the study was prematurely terminated due to moving to another place or pregnancy. The remaining 38 all had 4 time points (baseline, 1, 2, and 4 years), a total of 152 examinations. Examinations were performed during the daytime, between $8 \mathrm{AM}$ and 4 PM. Of these, 104 (68\%) were performed in the morning, and 48 $(32 \%)$, in the afternoon. The included subjects had a mean age of 


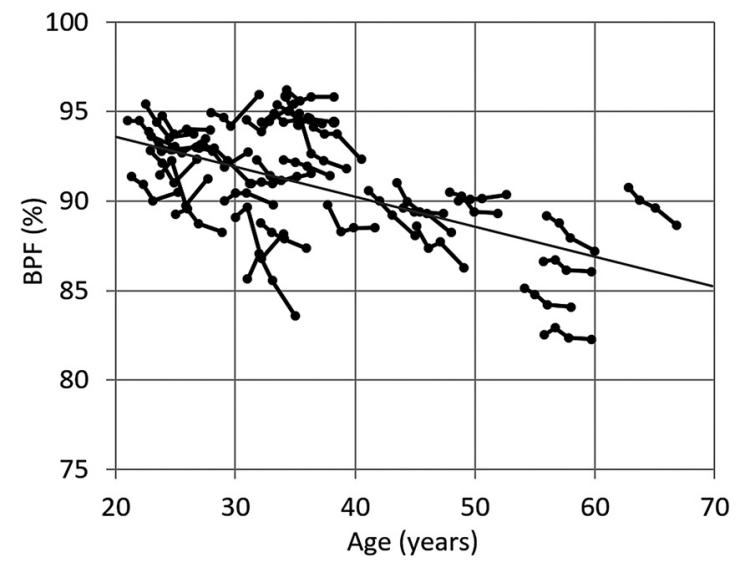

FIG 2. Brain parenchymal fraction of all subjects at all times. The 4 time points of each individual are connected with a line. The gray line indicates the linear regression slope.

$35 \pm 11$ years (range, 21-67 years) and a median Expanded Disability Status Scale of 2.0 (interquartile range, 1.0-2.0) at baseline. Further patient characteristics are presented in Table 1.

\section{Ethics Statement}

The study was approved by the regional ethics committee, and written informed consent was obtained from all participants.

\section{Acquisition}

The MR imaging quantification method was a multiecho, multidelay saturation recovery spin-echo sequence, which has been described previously. ${ }^{6}$ The multisection sequence consisted of section-selective saturation pulses, interleaved with a CarrPurcell-Meiboom-Gill acquisition of 5 echoes at multiples of 14-ms TE. The saturation pulse acts on a section $n$, while the subsequent acquisition acts on a section $m$. By a variable shift between sections $n$ and $m$, multiple effective delay times were created between the saturation and acquisition of each section. The sequence was repeated 4 times, resulting in a matrix of 20 images per section, at 5 different TEs and at 4 different saturation delay times. All these images had a different effect of $R_{1}$ and $R_{2}$ relaxation. The TR was $4280 \mathrm{~ms}$ with 43 sections of 3-mm thickness. The FOV was $230 \mathrm{~mm}$ with an in-plane resolution of 1.4 $\mathrm{mm}$. The scan time was 6 minutes and 20 seconds on an Achieva 1.5Tscanner (Philips Healthcare, Best, the Netherlands).

\section{Postprocessing}

The calculation of the intracranial volume (ICV), the brain parenchymal volume (BPV), and brain parenchymal fraction $(\mathrm{BPF}=\mathrm{BPV} / \mathrm{ICV})$ is an automatic function of SyMRI 9.0. The same software also shows the $R_{1}, R_{2}$, and PD maps of the entire acquisition volume. Typical images and maps from this software in our patients are shown in Fig 1. An adjustment was made in the software to extract the average $R_{1}, R_{2}$, and PD values for the entire brain and gray and white matter. This was done by taking the ICV and removing all CSF (clipped at 50\% partial volume) and subsequently eroding 1 pixel on all sides of the volume to remove the partial volume effects of CSF. The available GM and WM segmentation maps were used to retrieve the average $R_{1}, R_{2}$, and PD of GM and WM inside the eroded volume.
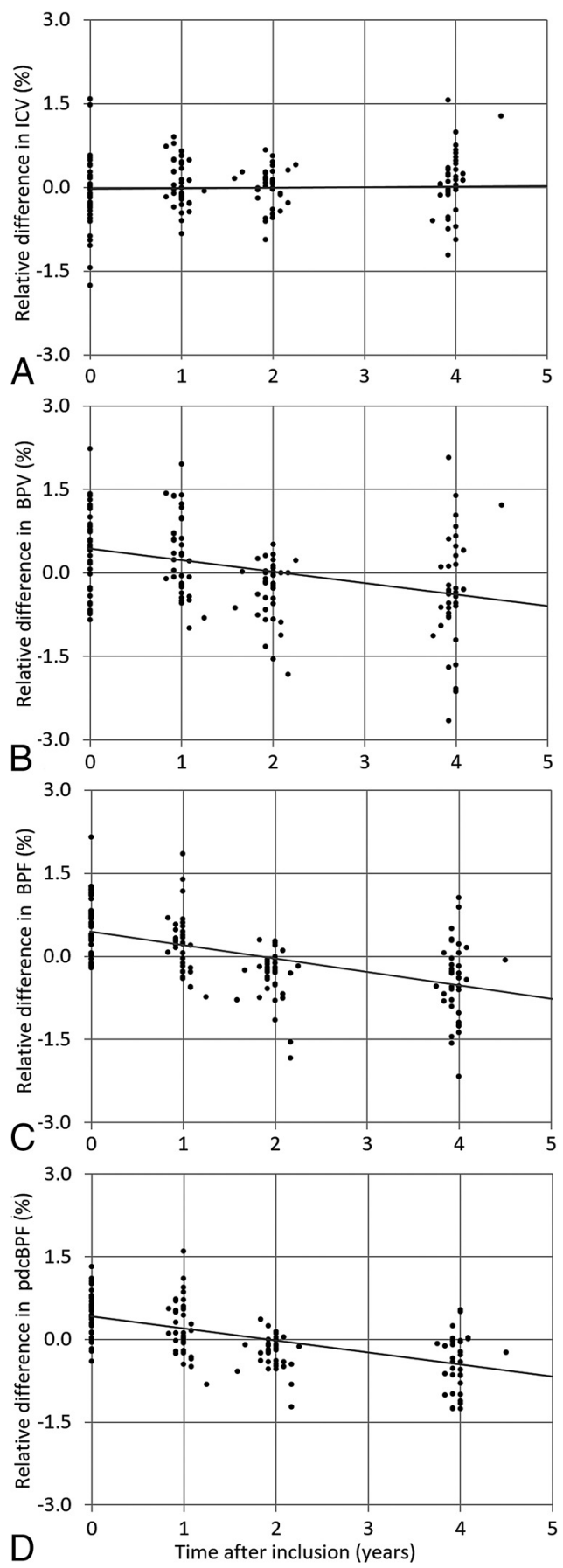

FIG 3. Relative difference of intracranial volume $(A)$, brain parenchymal volume $(B)$, and brain parenchymal fraction $(C)$ of all subjects as a function of time after inclusion of the study. $D$, The relative difference in BPF of all subjects is shown again as a function of time after inclusion is shown, but corrected for PD. This figure is comparable with $C$, albeit with less variation in the data. The gray lines indicate the linear regression slopes.

\section{Finding the Correlation of BPF with $R_{1}, R_{2}$, and PD}

The average value and SD of ICV, BPV, BPF, $R_{1}, R_{2}$, and PD were calculated for each time point. In the relative change of $\mathrm{BPF}_{\mathrm{i}}$ for all measurements, $i$ was found by dividing each of these measurements by the average $\langle\mathrm{BPF}\rangle_{\text {s }}$ of each subject $s$. This removed individual differences in BPF size. Subtraction by 1 centered the relative change of BPF around zero. The resulting data points 
Table 2: Observed correlation slopes $S_{R 1}, S_{R 2}$, and $S_{P D}$ of the relative change in BPF as a function of relative change in $R_{1}, R_{2}$, and $P D$ of the entire brain and gray and white matter

\begin{tabular}{lccc}
\hline & Brain & Gray Matter & White Matter \\
\hline $\mathrm{S}_{\mathrm{R} 1}$ value $(\% / \%)$ & -0.198 & -0.133 & -0.364 \\
$\mathrm{~S}_{\mathrm{R} 1}(95 \% \mathrm{Cl})(\% / \%)$ & $(-0.242$ to -0.152$)$ & $(-0.222$ to -0.044$)$ & $(-0.4376$ to -0.290$)$ \\
$\mathrm{S}_{\mathrm{R} 1} P$ value & $<.001$ & .02 & $<.001$ \\
$\mathrm{~S}_{\mathrm{R} 2}$ value $(\% / \%)$ & 0.156 & 0.303 & 0.090 \\
$\mathrm{~S}_{\mathrm{R} 2}(95 \% \mathrm{Cl})(\% / \%)$ & $(0.039-0.274)$ & $(0.228-0.378)$ & $(-0.016-0.196)$ \\
$\mathrm{S}_{\mathrm{R} 2} P$ value & .04 & $<.001$ & .2 \\
$\mathrm{~S}_{\mathrm{PD}}$ value $(\% / \%)$ & 0.488 & .396 & .655 \\
$\mathrm{~S}_{\mathrm{PD}}(95 \% \mathrm{Cl})(\% / \%)$ & $(0.382-0.594)$ & $(0.146-0.645)$ & $(0.535-0.776)$ \\
$\mathrm{S}_{\mathrm{PD}} P$ value & $<.001$ & .003 & $<.001$ \\
\hline
\end{tabular}
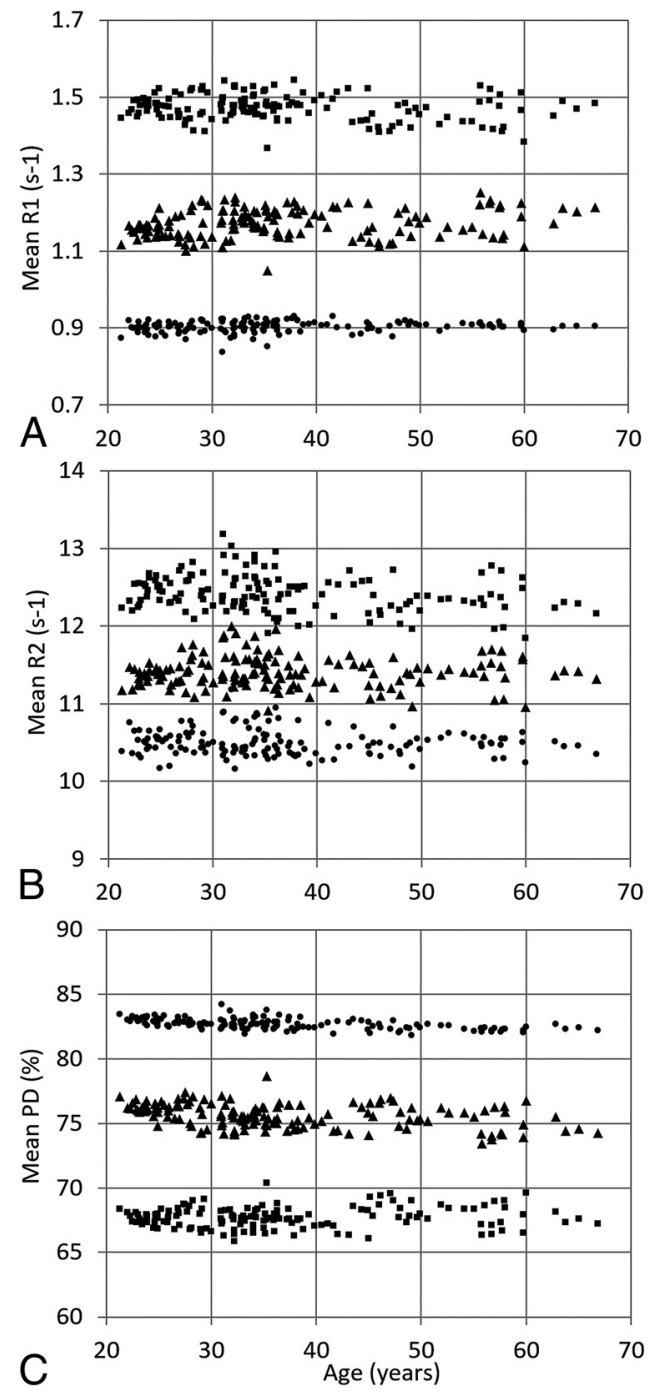

FIG 4. Observed mean $R_{1}(A)$, mean $R_{2}(B)$, and mean proton density (C) of the entire brain (triangles), gray matter (dots), and white matter (squares) as a function of age for all subjects.

were assumed to change linearly as a function of time after inclusion of $t$, expressed as an intercept and slope. A noise term $\varepsilon$ accounts for the observed variation of the data.

1) $\quad \frac{B P F_{\mathrm{i}}}{\langle B P F\rangle_{\mathrm{s}}}-1=$ Intercept + Slope $\times t+\varepsilon$.

We believe that the noise term $\varepsilon$ does not only contain random noise but may have a correlation with the observed relative change of $\mathrm{R}_{1}, \mathrm{R}_{2}$, and/or PD, independent of the time after inclusion. The relative change of $\mathrm{R}_{1}, \mathrm{R}_{2}$, and $\mathrm{PD}$ can be calculated in the same way as the relative change of BPF. Then, the correlation slopes $S_{R 1}, S_{R 2}$, and $\mathrm{S}_{\mathrm{PD}}$ were found according to

2) $\varepsilon=\left(\frac{B P F_{\mathrm{i}}}{\langle B P F\rangle_{\mathrm{s}}}-1\right)-$ Intercept

$$
\text { - Slope } \times t=S_{\mathrm{R} 1}\left(\frac{R 1_{\mathrm{i}}}{\langle R 1\rangle_{\mathrm{s}}}-1\right) \text {, }
$$

and equivalently for $\mathrm{R}_{2}$, and $\mathrm{PD}$.

\section{Correction of BPF}

Once the slopes $S_{R 1}, S_{R 2}$, and $S_{P D}$ are found, they can be used as correction terms for BPF to reduce $\varepsilon$. In other studies, it is unlikely that there is an average $R_{1}, R_{2}$, or PD per subject available; hence, the terms $\left\langle\mathrm{R}_{1}\right\rangle_{\mathrm{s}},\left\langle\mathrm{R}_{2}\right\rangle_{\mathrm{s}}$, and $\langle\mathrm{PD}\rangle_{\mathrm{s}}$ must be replaced by an expected $\mathrm{R}_{\text {lexp }}, \mathrm{R}_{2 \exp }$, and $\mathrm{PD}_{\exp }$ value, which can be taken from the average values of all our subjects. The corrected BPF is then calculated as the observed BPF multiplied by a factor $(1-\varepsilon)$.

\section{RESULTS}

\section{Finding the Correlation of BPF with $R_{1}, R_{2}$, and PD}

The average ICV of all subjects at all times was $1407 \pm 151 \mathrm{~mL}$; the average BPV was $1278 \pm 129 \mathrm{~mL}$, resulting in an average BPF of $90.9 \% \pm 3.2 \%$. The BPF of all subjects at all times is plotted in Fig 2. Linear regression on all data as a function of age showed a slope of BPF of $-0.167 \% /$ year ( $95 \%$ confidence interval, -0.131 to $-0.204 ; P<.001)$.

In Fig 3, the normalized ICV, BPV, and BPF are shown as a function of time after inclusion. For the separate time points, at 1,2 , and 4 years after the time of inclusion, the decrease of BPV and BPF was $2.2 \mathrm{~mL}(P=.4)$ and $0.37 \%(P<.001)$ after 1 year, $12.2 \mathrm{~mL}(P<.001)$ and $0.87 \%(P<.001)$ after 2 years, and $12.3 \mathrm{~mL}(P=.007)$ and $0.97 \%(P<.001)$ after 4 years. Linear regression as a function of all 4 years showed that the ICV had a nonsignificant slope of $0.02 \% /$ year ( $95 \%$ confidence interval, $-0.03-0.08 ; P=.6)$. The BPV showed a significant decrease with time after inclusion, with a slope of $-0.236 \%$ / year $(95 \% \mathrm{CI},-0.372$ to $-0.173 ; P<.001)$. Also, the BPF showed a significant decrease with time after inclusion, with a slope of $-0.273 \%$ /year $(95 \% \mathrm{CI},-0.211$ to -0.335 ; $P<.001)$. The intercept was $0.472 \%$.

The relative changes in BPF were corrected for time using the observed slope of $-0.273 \%$ /year and an intercept of $0.472 \%$ (Equation 2). Then, the resulting values for BPF were compared with the relative changes in the $\mathrm{R}_{1}, \mathrm{R}_{2}$, and $\mathrm{PD}$ to obtain the slopes $\mathrm{S}_{\mathrm{R} 1}, \mathrm{~S}_{\mathrm{R} 2}$, and $\mathrm{S}_{\mathrm{PD}}$. For $\mathrm{S}_{\mathrm{R} 1}$, a significant slope of -0.198 was observed $(P<.001)$. For $\mathrm{S}_{\mathrm{R} 2}$, this was $0.156(P=.04)$; and for $\mathrm{S}_{\mathrm{PD}}$, a significant slope of 0.488 was observed $(P<.001)$. Similar-butweaker correlations were found when only GM or WM were taken into account. All data are summarized in Table 2. No correlation was found between relative BPF and time of day of the examination $(P=.7)$. 
Table 3: Observed mean values and slopes of $R_{1}, R_{2}$, and PD of the entire brain and gray and white matter as a function of subject age

\begin{tabular}{lccc}
\hline & Brain & Gray Matter & White Matter \\
\hline $\mathrm{R}_{1}$ intercept $\left(\mathrm{s}^{-1}\right)$ & 1.151 & 0.897 & 1.495 \\
$\mathrm{R}_{1}$ slope $\left(\mathrm{s}^{-1} / \mathrm{y}\right)$ & 0.00054 & 0.00015 & -0.00063 \\
$\mathrm{R}_{1}$ slope $(95 \% \mathrm{Cl})\left(\mathrm{s}^{-1} / \mathrm{y}\right)$ & $(0.0002-0.0010)$ & $(-0.0001-0.0002)$ & $(-0.0011-0.0002)$ \\
$\mathrm{R}_{1}$ slope $P$ value & .04 & .4 & .2 \\
$\mathrm{R}_{2}$ intercept $\left(\mathrm{s}^{-1}\right)$ & 11.44 & 10.57 & 12.62 \\
$\mathrm{R}_{2}$ slope $\left(\mathrm{s}^{-1} / \mathrm{y}\right)$ & -0.0001 & -0.0018 & -0.0056 \\
$\mathrm{R}_{2}$ slope $(95 \% \mathrm{Cl})\left(\mathrm{s}^{-1} / \mathrm{y}\right)$ & $(-0.004-0.002)$ & $(-0.004-0.001)$ & $(-0.009-0.000)$ \\
$\mathrm{R}_{2}$ slope $P$ value & .5 & .3 & .05 \\
PD intercept $(\%)$ & 75.65 & 82.44 & 67.44 \\
PD slope $(\% / \mathrm{y})$ & -0.026 & -0.019 & 0.009 \\
PD slope $(95 \% \mathrm{Cl})(\% / \mathrm{y})$ & $(-0.039-0.013)$ & $(-0.025$ to -0.013$)$ & $(-0.003-0.020)$ \\
PD slope $P$ value & $<.001$ & $<.001$ & .2 \\
\hline Note:-y indicates year. & & &
\end{tabular}

Table 4: Observed SDs of the relative changes in BPF at baseline and at 1, 2, and 4 years after inclusion ${ }^{\mathrm{a}}$

\begin{tabular}{lcccc}
\hline & Baseline (\%) & 1 Year (\%) & 2 Years (\%) & 4 Years (\%) \\
\hline Uncorrected & 0.508 & 0.526 & 0.454 & 0.687 \\
Corr. for brain $\mathrm{R}_{1}$ & $0.362(28.8 \%)$ & $0.506(3.7 \%)$ & $0.327(28.1 \%)$ & $0.599(12.8 \%)$ \\
Corr. for brain PD & $0.385(24.2 \%)$ & $0.500(4.9 \%)$ & $0.303(33.3 \%)$ & $0.567(17.4 \%)$ \\
Corr. for GM R & $0.506(0.4 \%)$ & $0.465(11.5 \%)$ & $0.297(34.7 \%)$ & $0.561(16.2 \%)$ \\
Corr. for GM PD & $0.481(5.3 \%)$ & $0.539(-2.4 \%)$ & $0.398(12.5 \%)$ & $0.673(1.9 \%)$ \\
Corr. for WM R $R_{1}$ & $0.343(32.6 \%)$ & $0.463(12.0 \%)$ & $0.349(23.2 \%)$ & $0.568(17.3 \%)$ \\
Corr. for WM PD & $0.399(21.5 \%)$ & $0.474(9.8 \%)$ & $0.326(28.3 \%)$ & $0.492(28.3 \%)$ \\
\hline
\end{tabular}

Note:-Corr. indicates corrected.

a When BPF is corrected for average $R_{1}, R_{2}$, or PD values for brain, $G M$, or WM, the SDs of the observations decrease. The percentage reduction is given in parentheses. Corrections were only included when a high significance was found in Table 2

\section{Correction of BPF}

The observed mean $\mathrm{R}_{1}, \mathrm{R}_{2}$, and $\mathrm{PD}$ of the entire brain as a function of subject age are shown in Fig 4 (triangles). Only minor changes as a function of subject age were observed; linear regression showed a slope in $\mathrm{R}_{1}$ of 0.00054 seconds ${ }^{-1}$ /year. For $\mathrm{R}_{2}$, this was -0.0001 seconds $^{-1} /$ year; and for $\mathrm{PD}$, it was $-0.026 \%$ /year. The $\mathrm{R}_{1}$ intercept for all subjects was $1.151 \pm$ seconds ${ }^{-1}$, the $\mathrm{R}_{2}$ intercept was 11.44 seconds $^{-1}$, and the PD intercept was $75.65 \%$. All data, including for whole brain and GM and WM only, are summarized in Table 3. Using these values, one can establish the expected $R_{1}$ value for the whole brain of each subject, $R_{1 \exp }=1.151+0.00054 \times$ age; the expected $R_{2}$ value for the brain of for each subject, $\mathrm{R}_{2 \exp }=11.44-0.0001 \times$ age; and the expected $\mathrm{PD}$ value for the brain of each subject, $\mathrm{PD}_{\exp }=75.65-$ $0.026 \times$ age.

On the basis of these expected values for $R_{1}, R_{2}$, and $P D$, a correction of the BPF values can be made using Equation 2. The $\mathrm{PD}$-corrected $\mathrm{BPF}$ values, using $\mathrm{S}_{\mathrm{PD}}$, are shown in Fig $3 D$. In comparison with the uncorrected BPF values, as shown in Fig 3C, the variation of the data points was reduced. The SDs at baseline and at 1,2 , and 4 years after inclusion, when corrected for $\mathrm{R}_{1}, \mathrm{R}_{2}$, or $\mathrm{PD}$, are given in Table 4. The highest reductions were achieved with $\mathrm{R}_{1}$ and $\mathrm{PD}$, for which SDs decreased, on average, more than $20 \%$. The baseline values especially changed substantially. If one used the correction, the observed atrophy rate decreases slightly-for example, PD-corrected BPF shows a slope of $-0.238 \%$ /year, and $\mathrm{R}_{1}$-corrected $\mathrm{BPF}$ shows a slope of $-0.256 \%$ /year, decreases of $12.8 \%$ and $6.2 \%$, respectively, in comparison with the original slope of $-0.273 /$ year.

\section{DISCUSSION}

In this work, brain volume measurements were combined with measures reflecting the physical properties of the brain. Generally, the variation in brain volume measurements is high because it includes the normal variation in human head size. Thus, BPF is used for clinical follow-up because it normalizes brain volume with the intracranial volume, hence removing head size differences. ${ }^{17}$ An additional factor for brain volume variations is the placement of the MR imaging acquisition volume. The acquisition coverage in our case included 43 sections, with a total of $129 \mathrm{~mm}$, which was not sufficient for all subjects to cover the entire cranium. Differences of the placement of the acquisition volume may therefore have resulted in differences of the included ICV and BPV. This is a second reason that BPF is more reliable to use, because it is a ratio of the included volumes and hence less sensitive to the exact positioning of the acquisition volume. The stability of BPF over BPV is confirmed by the higher significance of the observed atrophy rate in our data.

Yet an additional factor, which is generally ignored, is that brain tissue composition may be different at each examination. Brain volume may not be as invariant as one may expect and may change with, for example, hydration, dehydration, time of day, blood pressure, physical activity, eating habits, or drug and alcohol consumption. Previous studies have shown that brain volume can exhibit a diurnal variation on the order of $0.4 \%-0.7 \%,{ }^{18,19}$ though this could not be verified in all studies. ${ }^{20}$ In our data, no evidence of a relation with the time of day was found either $(P=.7)$. Besides the general factors that can influence brain volume, patients with MS may exhibit additional effects. Starting treatment to suppress the inflammatory processes in the CNS is expected to reduce brain tissue edema, which hence may lead to an apparently accelerated brain atrophy directly after onset. The existence of the so-called pseudoatrophy has been described previously $y^{4,5}$ and is confirmed in, for example, natalizumab treatment, in which effective inflammation reduction leads to a higher initial brain volume loss, compared with the subsequent brain volume loss. ${ }^{21,22}$

For our study, we hypothesized that the average $\mathrm{R}_{1}$ and $\mathrm{R}_{2}$ and proton density of the brain were associated with water content of brain tissue, including the component due to edema. Our MR quantification approach has the advantage of simultaneously measuring $\mathrm{R}_{1}, \mathrm{R}_{2}$, and $\mathrm{PD}$ and automatically calculating brain volume and BPF using the same single sequence. We found that the average $R_{1}, R_{2}$, and PD values are relatively stable over the investigated age range. The total change of the observed values was $<2$ SDs during a complete lifetime (Fig 4). The mean values of WM and GM and the observed slow decrease of PD were very similar to previously reported values. ${ }^{23}$ 
A significant correlation was found between the relative change in observed BPF and the relative change in average $R_{1}$ and $\mathrm{PD}$ of the brain. No correlation was found for $\mathrm{R}_{2}$, in contrast to a previous study in which only $\mathrm{R}_{2}$ (T2) was used in a study with substantial dehydration and rehydration. ${ }^{24}$ The presence of these relations provides evidence that BPF is indeed affected by changes in brain tissue composition. The correlation is stronger in WM than in GM; this finding suggests that the average value for brain tissue is mainly affected by changes in WM for patients with MS. It means that when a subject exhibits a lower BPF, it is at least partly related to a higher $\mathrm{R}_{1}$ and a lower PD. In our study, no relation with the time of day was found, suggesting that the effect of disease activity is a stronger factor than diurnal fluctuations.

The observation that the variation in measured BPF can be reduced by correction of the average $\mathrm{PD}$ of the entire brain or the average PD of WM suggests that the level of edema in patients with MS varies, leading both to changes in total water content of brain parenchyma and changes in brain volume. Especially, the reduction of variation in BPF at baseline indicates that correction for edema could play an important role in the interpretation of the atrophy rate after the time of inclusion. If the effect of pseudoatrophy can be reduced, there would be no need to exclude an initial period of time to avoid the observation of an exaggerated atrophy rate, as was proposed as an alternative strategy. ${ }^{25}$ In our study, 13 patients had a clinical relapse within 2 months before MR imaging at baseline, compared with only 3, 1, and 2 at follow-up MR imaging. The higher proportion of patients in clinical relapse at baseline MR imaging was, in part, because the relapse was often the cause of neurologic evaluation and inclusion in the cohort study, in part because of a considerable number of patients receiving disease-modifying MS drugs during follow-up.

Limitations in our study were the low number of included patients, the specific selection of patients, and the inevitable differences in individual patient management, leading to additional factors that were treated as random variables in the analysis. The choice of a group of early-onset patients with MS precludes any general conclusions on patients with MS or other neurodegenerative diseases. No distinction was made using the Expanded Disability Status Scale because all patients had relatively low scores (interquartile Expanded Disability Status Scale range, 1.0-2.0). Also, no control group was included to assess the normal decrease of BPF or any correlation with $\mathrm{R}_{1}, \mathrm{R}_{2}$, or $\mathrm{PD}$ with the healthy brain, precluding a reference value for normal variation. The time between measurements was at least a year, which is too long to monitor the dynamic behavior of the disease activity. Because brain volume loss is a critical parameter in MS research and clinical management, however, this work must be seen as an indicator that brain volume measurements may be confounded by other factors and that the precision of such measurements may benefit from monitoring the brain tissue characteristics as well. Our study indicates that edema correction may improve the precision of monitoring brain volume loss for patients with MS. Possibly, other neurologic, psychiatric, and geriatric conditions could also benefit from such a correction, and we intend to include larger groups to investigate further possibilities. A faster rate of probing would potentially show disease dynamics, which would be interesting to investigate, especially in the first 12 months after onset, when the effect of pseudoatrophy is expected to predominate.

\section{CONCLUSIONS}

Edema correction for brain volume using the mean $\mathrm{R}_{1}$ and $\mathrm{R}_{2}$ or proton density of the brain reduced the variation in brain volume measurement values in our cohort with up to $33 \%$ and may have reduced the effect of pseudoatrophy.

Disclosures: J.B. Marcel Warntjes—UNRELATED: Employment: SyntheticMR AB, Comments: part-time employee; Stock/Stock Options: SyntheticMR AB. Peter Lundberg-UNRELATED: Employment: University Hospital; Grants/Grants Pending: Conventional National Science Council funding*. *Money paid to the institution.

\section{REFERENCES}

1. Vågberg $\mathrm{M}$, Lindqvist $\mathrm{T}$, Ambarki $\mathrm{K}$, et al. Automated determination of brain parenchymal fraction in multiple sclerosis. AJNR Am J Neuroradiol 2013;34:498-504 CrossRef Medline

2. Giovannoni G, Tomic D, Bright JR, et al. "No evident disease activity": the use of combined assessments in the management of patients with multiple sclerosis. Mult Scler 2017;23:1179-87 CrossRef Medline

3. ClinicalTrials.gov Identifiers: NCT01412333, NCT01247324, NCT02792218, NCT01226745, NCT02425644. www.clinicaltrials. gov. Accessed June 1, 2017

4. De Stefano N, Airas L, Grigoriadis N, et al. Clinical relevance of brain volume measures in multiple sclerosis. CNS Drugs 2014;28:147-56 CrossRef Medline

5. Zivadinov R, Reder AT, Filippi M, et al. Mechanisms of action of disease-modifying agents and brain volume changes in multiple sclerosis. Neurology 2008;71:136-44 CrossRef Medline

6. Warntjes JB, Leinhard OD, West J, et al. Rapid magnetic resonance quantification on the brain: optimization for clinical usage. Magn Reson Med 2008;60:320-29 CrossRef Medline

7. Warntjes M, Engström M, Tisell A, et al. Modeling the presence of myelin and edema in the brain based on multi-parametric quantitative MRI. Front Neurol 2016;7:16 CrossRef Medline

8. Kumar R, Delshad S, Woo MA, et al. Age-related regional brain T2-relaxation changes in healthy adults. J Magn Reson Imaging 2012; 35:300-08 CrossRef Medline

9. Neema M, Stankiewicz J, Arora A, et al. T1- and T2-based MRI measures of diffuse gray matter and white matter damage in patients with multiple sclerosis. J Neuroimaging 2007;17:16S-21S CrossRef Medline

10. Larsson HB, Frederiksen J, Petersen J, et al. Assessment of demyelination, edema, and gliosis by in-vivo determination of $\mathrm{T} 1$ and $\mathrm{T} 2$ in the brain of patients with acute attack of multiple sclerosis. J Magn Reson Med 1989;11:337-38 CrossRef Medline

11. Vymazal J, Righini A, Brooks RA, et al. T1 and T2 in the brain of healthy subjects, patients with Parkinson disease, and patients with multiple system atrophy: relation to iron content. Radiology 1999; 211:489-95 CrossRef Medline

12. Warntjes JB, Engström M, Tisell A, et al. Brain characterization using normalized quantitative magnetic resonance imaging. PLoS One 2013;8:e70864 CrossRef Medline

13. Hagiwara A, Warntjes M, Hori M, et al. SyMRI of the brain: rapid quantification of relaxation rates and proton density, with Synthetic MRI, automatic brain segmentation, and myelin measurement. Invest Radiol 2017;52:647-57 CrossRef Medline

14. Vågberg M, Ambarki K, Lindqvist $\mathrm{T}$, et al. Brain parenchymal fraction in an age-stratified healthy population: determined by MRI using manual segmentation and three automated segmentation methods. J Neuroradiol 2016;43:384-91 CrossRef Medline

15. Granberg T, Uppman M, Hashim F, et al. Clinical feasibility of Syn- 
thetic MRI in multiple sclerosis: a diagnostic and volumetric validation study. AJNR Am J Neuroradiol 2016;37:1023-29 CrossRef Medline

16. Polman $\mathrm{CH}$, Reingold SC, Banwell B, et al. Diagnostic criteria for multiple sclerosis: 2010 revisions to the McDonald criteria. Ann Neurol 2011;69:292-302 CrossRef Medline

17. Rudick RA, Fisher E, Lee JC, et al. Use of the brain parenchymal fraction to measure whole brain atrophy in relapsing-remitting MS: Multiple Sclerosis Collaborative Research Group. Neurology 1999;53:1698-704 CrossRef Medline

18. Nakamura K, Brown RA, Narayanan S, et al. Diurnal fluctuations in brain volume: statistical analyses of MRI from large populations. Neuroimage 2015;118:126-32 CrossRef Medline

19. Duning T, Kloska S, Steinsträter, et al. Dehydration confounds the assessment of brain atrophy. Neurology 2005;64:548-50 CrossRef Medline

20. Meyers SM, Tam R, Lee JS, et al. Does hydration status affect MRI measures of brain volume or water content? J Magn Reson Imaging 2016;44:296-304 CrossRef Medline

21. Magraner M, Coret F, Casanova B. The relationship between inflam- matory activity and brain atrophy in natalizumab treated patients. Eur J Radiol 2012;81:3485-90 CrossRef Medline

22. Mellergård J, Tisell A, Blystad I, et al. Cerebrospinal fluid levels of neurofilament and tau correlate with brain atrophy in natalizumab-treated multiple sclerosis. Eur J Neurol 2017;24:112-21 CrossRef Medline

23. Neeb H, Zilles K, Shah NJ. Fully-automated detection of cerebral water content changes: study of age- and gender-related $\mathrm{H} 2 \mathrm{O}$ patterns with quantitative MRI. Neuroimage 2006;29:910-22 CrossRef Medline

24. Nakamura K, Brown RA, Araujo D, et al. Correlation between brain volume change and $\mathrm{T} 2$ relaxation time induced by dehydration and rehydration: implications for monitoring atrophy in clinical studies. Neuroimage Clin 2014;6:166-70 CrossRef Medline

25. De Stefano N, Giorgio A, Battaglini M, et al. Reduced brain atrophy rates are associated with lower risk of disability progression in patients with relapsing multiple sclerosis treated with cladribine tablets. Mult Scler 2017 Jan 1. [Epub ahead of print] CrossRef Medline 